\title{
Establishing the Hallmarks of a Convincing Chatbot-Human Dialogue
}

\author{
Jurek Kirakowski, Patrick O'Donnell and Anthony Yiu \\ Human Factor Research Group, UCC, Cork Enterprise Centre \\ Cork, Ireland.
}

\section{Introduction}

Intelligent agents are systems that are capable of performing actions on behalf of computer users; in essence, reducing the cognitive workload on users who are engaging with computer systems. There is a potentially diverse range of applications for such agents in software use. These types of agents have a part to play in domains such as user interfaces, the negotiation of information retrieval and organization, and electronic purchasing. Intelligent agents represent the active use of weak Artificial Intelligence units in computer use in general. The search engine "Encarta", devised by Microsoft, is a good example which demonstrates some of the applications associated with these kinds of agents. The software uses Natural Language Processing (NLP) in order to give the program ability to carry out sophisticated information retrieval tasks. This stems from the capacity of sophisticated NLP programs to parse the typed requests of human users and approach an "understanding" of these requests in terms of keywords which can then be used to search the web for relevant information and parse the resulting text in order to construct a response to a request.

"Encarta" offers an attractive user interface because it can parse, analyse and produce natural language utterances. This allows the program to produce relevant responses to human utterances in natural language form. As such, it represents a particular style of intelligent agent, most commonly known as a Chatbot (or Chatterbot). It is this aspect of NLP agents as Chatbots that is the concern of this paper. In theory, a powerful enough Chatbot could offer a hugely attractive form of Human Computer Interaction in that computer use could be mediated by an agent that behaves as though it can understand instructions that are typed in by human users and respond in kind with natural-seeming utterances. The point has been raised that in order to communicate with computers, humans must learn the language of computers and that computers are, at present, incapable of communicating by using human languages (Pinker: 2004). The prospect of an Chatbot that is powerful enough to deal with human languages would radically change this state of affairs.

There are several ways of approaching the problem of building a software engine that has to deal with human language utterances. Since an agent that is successful in this task is essentially a form of Artificial Intelligence, it seems fitting to begin by describing the 
traditional approach to AI and how it relates to this problem. Traditional AI approaches rely on a Strong Physical Symbols System approach (SPSS), whereby a series of symbols is given to the engine in question, the symbols are manipulated in some logical manner within the engine and a series of symbols is given as output (Newell and Simon; 1976). For language systems such as Chatbots, this would involve arming the program with certain rules about grammar and word meanings. The system relies on manipulating these rules to derive meaning from incoming information and to then construct responses. If for example, a Chatbot built using the SPSS approach were given an input such as "The dog was big", it would decompose the sentence into its component parts; 'The' (determiner) 'dog' (noun) 'was' (verb) 'big' (adjective) and then ascribe a meaning to each component of the sentence, perhaps from a database of related concepts ('big' being an adjective related to size). The advantage of this approach to language processing is that, once a sentence such as 'The dog was big' can be analysed, any sentence employing a similar structure ('A girl will arrive') can be similarly interpreted, since the same grammar rules are involved in examining this sentence structure.

There are however several problems attendant on these approaches, both in general and in the specific case of language processing. A general criticism of SPSS approaches is that of symbol grounding (Harnad; 1990). The problem is essentially that of how a system that depends on the manipulation of virtual symbols can ever ascribe any kind of meaning to those symbols, except in terms of other arbitrary symbols thus defined themselves. So, even though a Chatbot system is able to deduce that 'big' is an adjective related to 'size', it will have no essential understanding of the concept 'big' since it has no essential understanding of a concept like 'size'. There is no way in which a system can arrive at an understanding of concepts by simply relating them to other concepts.

Another problem that is of particular interest with regards SPSS-style Chatbots in as far as they approach language processing is the problem of emergent problem spaces, effectively, the tendency of such systems to generate all possible meanings and interpretations of a sentence that are allowed by the grammar of a language, without being able to settle on one appropriate interpretation. This problem is endemic of all SPSS systems, but is especially relevant to language processing as it is a task that must occur in real time, and any attempt by a language engine to test each individual solution before choosing the correct one will be time consuming. Indeed, even if the time taken to process all possible variations in the meaning of a sentence were not an issue, it is likely that SPSS style Chatbots would never be able to settle on a suitable meaning for a sentence since, as discussed, they have no essential understanding of the various concepts that might be represented in the sentence and thus cannot relate those concepts to non-verbal, contextual cues. The classic example of this type of problem in language processing comes from early attempts to build sentence parsers along this line. The engine, when tested, generated five possible meanings for the sentence "Time flies like an arrow." (Bobrow: 1963). These alternate interpretations of the sentence involved the system ascribing different meanings to concepts like 'time' and 'arrow'. Thus, while the most obvious way of interpreting this sentence for most people would be that 'time moves quickly, like an arrow', the Chatbot engine also generated interpretations such as 'a species of fly, known as the "time fly" enjoys arrows'. 
The second prominent approach in contemporary Artificial Intelligence is the connectionist approach (also referred to as parallel distributed processing or the neural net approach). This approach operates in a fundamentally different way to SPSS style processing. Connectionist approaches eschew strong, inviolable rules in favour of a network of concepts that are related to one another to greater or lesser extents. The degree of relatedness between two concepts is flexible. There are at least three unique features that make a connectionist network a powerful system for handling human conversation: namely superpositioning, intrinsic context sensitivity, and strong representational change. (Clark 1993)

Firstly, two representations are said to be fully superposed when the resources used to represent item 1 are co-extensive with those used to represent item 2 . The natural mechanism of connectionist learning and superpositioning-storage yield a system that will extract the statistical central tendency of an exemplar. This is usefully seen as embodying prototype-style knowledge representation.. The network extracts various feature complexes and thus comes to encode information not just about specific exemplars but also about the stereotypical features set displayed in the training data. In other words, a number of concepts are related to one another in the connectionist system in order to yield the prototypical example of a class or category. For a concept like 'dog', sub-concepts like 'fourlegged', 'hairy' and 'animal' would be related to each other to produce an archetypal example of 'dog' The network can generalise novel cases sensibly by dint of its past training.

Secondly, the connectionist network concept can also display intrinsic context sensitivity. The most radical description of this would be that connectionist system does not involve computations defined over symbols. Instead, any accurate picture of the system's processing will have to be given at the numerical level of unites, weights and activation-evolution equations, while the symbolic-manipulating computational description will at most provide a rough guide to the main trends in the global behaviour of the system (Clark, 1993). The network can then learn to treat several inputs, which result in subtly different representational states, as prompting outputs which have much in common. So, many inputs may have features in common with a prototypical representation of a concept, but also have distinct differences (a three-legged dog, for example). In these cases, the network is capable of adjusting to the fact that slight differences in input do not necessitate a radically new concept (a whole new category for 'dog' that deals only with three-legged dogs, say).

Thirdly, a connectionist network can show strong representational change. Fodor (1981) suggested concept learning can only consist in the triggering of innate representational atoms or the deployment of such atoms in a "generate and test" learning style. According to Clark (1993) this is weak representational change as the product necessarily falls within the expressive scope of the original representational base. The connectionist network on the other hand, can acquire knowledge without the benefit of any such resource. For example the NETtalk (Sejnowski and Rosenberg 1986) and the past-tense learning network (Rumelhart and McClelland 1986) both begin with a set of random connection weights and learn about a domain "from scratch". Such adaptiveness is a vital feature of any system that must engage in processing language, since language is, by its nature, a fluid and changing system of rules and related meanings. 
Thus, Connectionism has much to recommend it; its sensitivity to contextual change makes Chatbot-systems based on connectionist models extremely adept at picking up on fine shades of meaning. A connectionist-style Chatbot would be much less likely to be caught between five alternative meanings of a sentence like 'time flies like an arrow' than would an SPSS-style system.

Connectionist models however have a similar symbol-grounding problem as the SPSS approach does. Connectionist models explain symbols by a series of context-sensitive connections. The process itself does not 'bottom-out' or come to a definition that is not prone to context infection. In other words, even if a connectionist system is capable of dealing in finer shades of meaning than an SPSS-style system, there is no point at which the small, atomic concepts that go into building up bigger concepts are related to the world at large in any essential way. In additional, there is a problem involving systematicity (Hadley, 1994) in that a connectionist network can fail to process sentences with constituents in novel syntactic position and at a novel level of embedding when processing includes determining the word's semantic role.

The symbol grounding problem is the problem of representing meaning in a system of purely arbitrary symbols. One approach to robotics and AI in general that may be able to address this problem involves dodging the question of representation altogether. Wallis (2004) discusses the possibility of producing systems that can exhibit all the characteristics of an intelligent agent (intention, planning etc.) without using any more representation "than a microwave oven would." Wallis' stance is informed by Brooks' (1991) approach to robotics, wherein robots can be developed that behave in an autonomous, intelligent fashion without any bona fide "understanding" of their own behaviours or why they are performing them. The essential tenet that underlies this approach is that an agent's intelligent behaviour arises out of an interaction between the agent and its environment, in the service of achieving some goal. Reflective reasoning about the environment, the goal or the behaviour by the agent is not necessary for the behaviour to be described as intelligent. The agents that exhibit this type of architecture can perform behaviours that can be described as intelligent without possessing any capacities that we would describe as "intelligence" because their actions make sense within their environment with regards to the satisfaction of some goal. Thus, if it is possible to produce a chatbot that has no representation of meaning but can behave as though it did (i.e. seem to understand utterances and interact with users), then users would be forced to conclude that its conduct within a dialogue was "intelligent". The earliest types of chatbot programs, that scan for keywords and match responses, can be seen as non-representational chatbot agents. The archetypal example of this type of Chatbot is 'Eliza', a system that consists of a database of phrases to be matched to keywords. The Chatbot itself does not contain anything that we might label as an 'understanding' of the concepts that it is discussing or the process by which it operates, but users can be gulled into believing (if only for a short period of time) that the Chatbot with whom they are communicating is in fact a person. Essentially, if the ability of non-representational Chatbots like these ones can be extended, to have users fully or partially convinced that they are conversing with another human being, then to all intents and purposes we can say that we have an 'intelligent language-processing system'. It is intended to specifically examine the way in which one of these non-representational Chatbot 
agents will interact with users, and how it might be possible to improve on its ability to be regarded as an agent that produces intelligent language behaviour. What is interesting is whether a non-representational approach such as this can be brought to bear in an arena such as language, a system of representational symbols.

It is not appropriate in this paper to attempt to select between these three approaches to the architecture of a natural language machine. No doubt in the end the "best" approach will be a hybrid of some kind and there are problems of principle as well as of implementation. We note that in the past research has taken a particular technology as a given and focussed on the application. We propose to turn the problem round.

That is, it is intended to do a much more "requirements" orientated survey, to identify what aspects of language comprehension and production by software agents characterise them as being "inhuman" in the eyes of computer users and which aspects are characteristic of human language behaviour. It may later be appropriate to discuss which types of programs and architectures are best equipped to support the type of behaviours seen as quintessentially "human". In other words, the research question addressed in this paper is: when users interact with an agent that is equipped to process human language and respond with utterances of its own, what kind of mistakes can the program make that a human wouldn't and that make the dialogue between user and agent seem unnatural. At that point, it will perhaps be more appropriate to return to questions of which approach to Artificial Intelligence is best equipped to deal with the various problems involved in language processing.

\section{Method}

In the experiment fourteen participants were asked to interact with an Eliza-style computer program (chatbot) for three minutes and then to participate in the elicitation of critical incidents with a transcript of their session. The program was based on the classic Eliza design with two important differences. Firstly, there was no mechanism that retained previous phrases entered by the user which could be used to re-start a stalled conversation (eg: "Tell more more about [a previous utterance]".) This was for theoretical reasons as will be discussed later. Secondly, there was a mechanism which enabled the chatbot to switch contexts on detecting particular words. Thus if the chatbot detected the word "music" the whole list of trigger phrases and responses changed to a music-orientated set.

A qualitative approach incorporating the Critical Incidents Technique (CIT) and content analysis of responses is involved in this study. At the end of this interaction, the participants were presented with a printed transcript of the dialogue and asked to highlight instances of the conversation that seemed particularly unnatural (up to three examples) and then to report why this was so. The same was done for up to three examples of speech that did seem convincing. The data produced by the critical incident technique were content analysed, with user responses being sorted by theme. The data coding was cross-checked independently by another individual. Inter-rater reliability of approx. 0.53 was obtained in the first pass. Items on which there was disagreement were discussed and placed in mutually agreeable categories with the assistance of a third independent rater. We are 
reasonably sure that the categories that have emerged represent reproducible aspects of the data set.

\section{Results}

The various themes that were produced during the content analysis were as follows. Firstly, under the heading of unconvincing characteristics-

$>$ Fails to maintain a theme once initiated. In that, once a theme emerged in the dialogue, the chatbot failed to produce statements relevant to that theme in the following section of the dialogue.

$>$ Formal or unusual treatment of language. Some statements in the chatbots database seemed overly stiff and formal or used unusual words and language.

$>$ Failure to respond to a specific question. Users would ask for a specific piece of information, such as asking the chatbot what its favourite film might be, and receiving no answer.

$>$ Fails to respond to a general question or implicit cue. Users offer the chatbot a cue (in the form of a general question, like "How are you?", or offer a cue in the form of a statement, like "Tell me about yourself." Or "Let's talk about films then.") and receive an irrelevant response.

$>$ Time delay. A fairly cosmetic fault, users felt that the chatbot responded too quickly to a detailed question or too slowly to a courtesy.

$>$ Phrases delivered at inappropriate times, with no reference to preceding dialogue. Where generic type phrases did not fit into the conversation in a natural way, or the chatbot responds to an inappropriate key phrase, with a resulting nonsequitur.

Under the heading of convincing aspects of the conversation-

$>$ Greetings. Several participants identified the greeting as a human-seeming characteristic.

> Maintains a theme. When the chatbot introduced a theme and was successful at producing a few statements that were relevant to that theme, users found this convincing.

$>$ Damage control. When the chatbot produced a breakdown in communication (for any of the reasons mentioned earlier) and then produced a statement that seemed to apologise for the breakdown or seemed to redirect the conversation in a more fruitful direction, users found this a convincingly human trait.

$>$ Reacts appropriately to cue. Users found it convincing when the chatbot responded appropriately to a cue such as "How are you?" or "Tell me about yourself."

$>$ Offers a cue. Users found it convincing when the chatbot offered a cue for further discussion, such as "What do you want to talk about?" or offered a range of topics for discussion.

$>$ Language style. Users found conversational or colloquial English to be convincing.

$>$ Personality. The fact that the chatbot was given a name (in fact, even users who did not report the inclusion of a name as convincing referred to it as a "Sam" or a "he") suggests that users wish to assign a personal agency to the chatbot even in the teeth of discrepant knowledge. 


\section{Discussion}

This research focuses on requirement and not any kind of implementation. For now it is enough to identify what traits in the bot-human interaction make it different to humanhuman interaction and how best these shortcomings might be addressed. Indeed, a reassuring symmetry emerges in the themes identified by users as being convincing or not: maintaining a theme is convincing, while failure to do so is unconvincing, formal or unusual language is unconvincing while colloquial or conversational English is the opposite. Reacting appropriately to a cue is human while failing to a react to one isn't. Delivering an unexpected phrase at an inappropriate time does not impress, but damage control statements can rectify the situation. It is time to address each feature of the bot-human dialogue in a little more detail.

\section{Maintenance of themes}

One of the factors, upon which the success or failure of the program to appear human seems to depend, is its ability (or lack thereof) to maintain a conversational theme once introduced. The Eliza-style chatbot used in this trial has no memory of a conversation as such (it operates on a first order Markov process, whereby each token is generated in response to the token immediately preceding, with no reference to the accumulated tokens, in this case token $=$ utterance and accumulated tokens $=$ the whole dialogue). This does not preclude it from maintaining a theme however; indeed several participants reported its ability to do so as a convincing feature of its dialogue. The means by which this is accomplished (given that the program has no "memory" of the conversation) is now described.

The chatbot used was unlike the classic Eliza program in that as well as having specific phrases activated by the presence of a keyword, the program could activate a whole database of phrases in response to a key phrase that are specifically related to that phrase (for example, an inventory of keyword-response pairs that are related to music can be prompted by the word "music").

Thus, the program has access to a database of phrases that are most likely to be relevant to the theme raised. At present, failure to maintain a theme that has activated one of these databases may be due to the fact that these databases contain all the same generic response phrases and keyword-response pairs as the general text database that serves as the default set of responses. This makes the likelihood that a theme-relevant phrase is activated lower than if the specialised databases were to contain theme-relevant phrases only. Thus, a means of improving the ability of this program to maintain a theme in conversation might be to enlarge the number of theme relevant keyword-response pairs in these databases and remove most of the generic keyword-response pairs from these "themed" databases.

\section{Failure to respond to a specific question}

This problem, essentially, is a question of how much information is contained in the program's memory and whether or not it can be accessed. Thus, if a person were to ask the program "What is the capital of France?" and the program did not have the information required, the program seems less human. There is no easy way to solve this problem. The 
solutions are to give the program a large enough database of information to be able to cope with most information requests of this kind (this approach suffers from the fact that the database is still a finite resource and almost certainly contains less information than a human would be expected to) or to grant the program access to the internet and equip it with a more powerful means of parsing information requests so that it can then establish the exact nature of a request and search for the relevant data on the internet. This first solution is brute force and is probably most relevant to a personal-use "humanised" AI, with a role as a user-interface for small-scale personal computer use, while the second is the type of approach that might be associated with a general information retrieval agent such as Microsoft's "Encarta".

\section{Responding to social cues}

This category covers the failure or success of the program to react appropriately to a social cue such as "How are you?" or "Tell me about yourself." Some of these cues can be treated in a similar way to the information requests dealt with above, in that an appropriate response can be matched, from a database, to a specific cue.

\section{Formal and colloquial language}

In general, formal language was regarded as being an unconvincing trait of the program's, with casual or colloquial language being preferred. Replacing formal phrasings with casual equivalents is a relatively minor adjustment that can be made to improve the program's performance. It is worth bearing in mind however that this trial involved a chatbot that was geared towards free conversation as opposed to being a helper agent in a structured task. In other circumstances, language style might not be a consideration for users at all, or perhaps even more formal and precise language might be preferred (eg in making a financial transaction.)

\section{Greetings and personality}

Some users reported that certain surface details involved in the chatbot's dialogue made it seem more human by their very presence. The fact that the bot "introduced itself" at the beginning of the dialogue and was given a human name for the trial influenced people into regarding it as slightly more human. This is separate from the functional issues involved in recognizing conversational breakdown and issuing damage repair, and is probably more related to personal preferences.

\section{Offers a cue}

The chatbot was deemed to be very "humanlike" when it offered cues on which users could elaborate. The possibility has already been raised of including more cues which are designed to elicit clarification in situations where the chatbot does not have enough information to respond appropriately to a cue. This promotes information exchange between the user and the chatbot and is likely to reduce ambiguity and allow the chatbot to react more reliably to user-statements. 


\section{Phrases delivered at inappropriate times}

This is an enduring problem of the Eliza style keyword-response chatbot, generic phrases are produced which do not fit well into the conversation, or a keyword prompts a response that is inappropriate in the context it is used. The first problem can be caused when the generic "placeholder" phrase is a poor one. In the case of the second problem, the chatbot might produce an inappropriate phrase due to the fact that it is insensitive to context. A word which means one thing in a certain context, and which prompts an appropriate response, might mean something completely different in a different context and the same response, when prompted, will no longer be appropriate. Some suggestions for remedying this problem are to equip the program with statements that ask for clarification and to refine the types of keywords that prompt particular responses. In addition, a chatbot that relies on a connectionist architecture may well be more sensitive to context than the model described here and may thus be able to select appropriate responses with a high degree of accuracy.

\section{Damage Control}

In certain situations, the chatbot seemed to be offering to change the topic of conversation after a particular line of conversation broke down, or to try and clarify previous statements. This is a further example of the kind of information-exchange that can occur between users and agents. Not only does this ability seem to make the chatbot appear more human, it would be a valuable ability to develop in any of the major potential applications of chatbots as helpful agents. This type of capability would allow for a more refined search when using information-retrieval agents. In personal computer user-interfaces, this kind of informationexchange opens up the possibility for the agent to make suggestions as regards computeruse.

\section{Comments on method of analysis}

With regards to the method of analysis employed in this study, it is intended to discuss the level to which the Critical Incident Technique was an appropriate tool of assessment in this trial. The benefits of the Critical Incident Technique as regards this study were as follows:

$>$ Rare events were noted as well as common events, thus the situation in which bothuman interaction could break down and then be retrieved by the bot in a damage control exercise did not occur in all or most of the dialogues but it was identified alongside more common shortcomings of the bot nonetheless.

Users were asked to focus on specific instances of communication breakdown (as opposed to being allowed to offer the vague opinion that the dialogue "felt wrong") and this allows for a more precise focus on individual problem areas (such as being able to treat "failure to answer a specific question" as a separate problem to "failure to respond to a general question or cue").

However, some shortcomings of the Critical Incident Technique as used in this trial were as follows:

There is no indication as to the relative severity of failures by the bot to appear human. In other words, it is difficult to tell if users found the agent's inability to 
maintain a conversational theme a more serious problem than the delivery of unexpected and inappropriate phrases during the dialogue, or even if there is a degree of individual difference involved in which characteristics of the bot's conversation-style are pertinent to its seeming human.

$>$ This method of analysis requires a focus on specific incidents of success or failure and is not particularly sensitive to context. This trial involved a simulated conversation, in which context would be important in establishing whether or not the dialogue seemed natural and though participants are asked to describe the events that lead up to a critical incident as part of their report, some information regarding the context of the conversation as a whole is probably missed.

\section{References}

Bobrow, D: Syntactic Analysis of English by Computer - A Survey, tech report 1055, BBN 1963

Brooks, R.A.: Intelligence without representation, Artificial Intelligence 47 (1991), 139-159.

Clark, A.: Associative Engine: Connectionism, Concepts, and Representational change. Bradford Book; London, England (1993)

Fodor, J: Representations: Philosophical Essays on the Foundations of Cognitive Science. MIT Press. (1981)

Hadley, R. F: Systematicity in connectionist language learning. Mind and Language, Vol. 9 P.247-272. (1994)

Harnad, S: The Symbol Grounding Problem. Physica D 42: 335-346. (1990)

Newell, A., and H. A. Simon: Computer science as empirical inquiry: Symbols and search. Commun. Assoc. Comput. Machinery 19: (1976) 111-26.

Pinker, S: The Language Instinct. Penguin. London (1994) p193

Rumelhart, D. and McClelland, J:`On learning the past tense of English verbs' in Parallel Distributed Processing: Exploration in the Microstructure of Cognition, Vol. 2, ed. Rumehart et al. MIT Press (1986)

Sejnowski, T., and Rosenberg, C: NETtalk: A Parallel Network That Learns to Read Aloud. Technical report JHU/ECC-86/01, John Hopkins University. (1986)

Wallis P., Intention without representation, Philosophical Psychologyy, vol. 17, no. 2 (2004) 


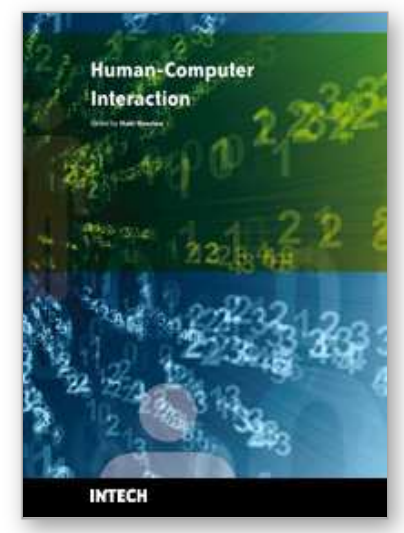

\section{Human-Computer Interaction \\ Edited by Inaki Maurtua}

ISBN 978-953-307-022-3

Hard cover, 560 pages

Publisher InTech

Published online 01, December, 2009

Published in print edition December, 2009

In this book the reader will find a collection of 31 papers presenting different facets of Human Computer Interaction, the result of research projects and experiments as well as new approaches to design user interfaces. The book is organized according to the following main topics in a sequential order: new interaction paradigms, multimodality, usability studies on several interaction mechanisms, human factors, universal design and development methodologies and tools.

\section{How to reference}

In order to correctly reference this scholarly work, feel free to copy and paste the following:

Jurek Kirakowski, Patrick Oâ€TMDonnell and Anthony Yiu (2009). Establishing the Hallmarks of a Convincing Chatbot-Human Dialogue, Human-Computer Interaction, Inaki Maurtua (Ed.), ISBN: 978-953-307-022-3, InTech, Available from: http://www.intechopen.com/books/human-computer-interaction/establishing-thehallmarks-of-a-convincing-chatbot-human-dialogue

\section{INTECH}

open science | open minds

\section{InTech Europe}

University Campus STeP Ri Slavka Krautzeka 83/A 51000 Rijeka, Croatia Phone: +385 (51) 770447

Fax: +385 (51) 686166 www.intechopen.com

\section{InTech China}

Unit 405, Office Block, Hotel Equatorial Shanghai No.65, Yan An Road (West), Shanghai, 200040, China 中国上海市延安西路65号上海国际贵都大饭店办公楼405单元 Phone: +86-21-62489820

Fax: +86-21-62489821 
(C) 2009 The Author(s). Licensee IntechOpen. This chapter is distributed under the terms of the Creative Commons Attribution-NonCommercial-ShareAlike-3.0 License, which permits use, distribution and reproduction for non-commercial purposes, provided the original is properly cited and derivative works building on this content are distributed under the same license. 